\title{
Eficacia simbólica de la ley 1620 del 2013 sobre Sistema de Convivencia Escolar ${ }^{1}$
}

\author{
Symbolic effectiveness of law 1620 / 2013 about School Coexistence System. \\ Leonel Castro Herrera² \\ RESUMEN
}

La Ley 1620 de 2013 creó el Sistema Nacional de Convivencia Escolar y Formación para el ejercicio de los derechos humanos, sexuales y reproductivos, reglamentada por el Decreto 1965 de esa misma anualidad. Ahora bien, el objeto central de esta disertación es profundizar las problemáticas tan complejas que pretende regular dicha norma, comprender los elementos que componen este sistema político-jurídico, establecer la efectividad de sus objetivos explícitos y analizar la eficacia simbólica de su promulgación.

Palabras claves: Convivencia escolar, eficacia simbólica, derechos humanos, sexuales y reproductivos.

\begin{abstract}
The Law 1620 of 2013 created the National System of School Coexistence and Training for the exercise of human, sexual and reproductive rights, regulated by Decree 1965 of that same annuity. Now, the central object of this dissertation is to deepen the complex problems that the regulation aims to regulate, to understand the elements that make up this political-juridical system, to establish the effectiveness of its explicit objectives and to analyze the symbolic efficacy of its promulgation.

Keywords: School coexistence, Symbolic effectiveness, Human, sexual and reproductive rights.

\footnotetext{
1El presente escrito es un avance del proyecto de investigación denominado “Escuela de formación ciudadana, derechos humanos y convivencia escolar", avalado y financiado por Colciencias (2015-2016). Para la realización del mismo se empleó el método de investigación documental, que supuso una serie de lecturas de documentos bibliográficos, hemerográficos, académicos e institucionales sobre el acoso escolar y otras formas de violencia que afectan a los niños, niñas y adolescentes en Colombia y en el mundo, así como un análisis en torno la noción de eficacia simbólica desarrollada por el profesor Mauricio García Villegas.

2 Abogado de la Universidad del Atlántico, Investigador del Grupo Cronotopías, Maestrando en Derecho de la Universidad Nacional de Colombia. Autor de diversos artículos y capítulos de libro sobre teoría política, derecho e interculturalidad publicados en revistas y libros de alcance nacional e internacional. Email: leonelcastroherrera@gmail.com
}

Recibido: 17 de Septiembre de 2018

Aprobado: 7 de Noviembre de 2018.

Revista Legem, Universidad del Atlántico 4 (2), Julio - Diciembre 2018, pp. 21-51 ISSN: 2346-2787 


\section{Introducción}

En primer lugar, nos encontramos en un momento histórico ante la inminente posibilidad de que se concreten e implementen los acuerdos de paz entre el Gobierno de Colombia, las FARC y un eventual acuerdo con el ELN, lo cual supone una serie de retos para el Sistema de Convivencia Escolar, no sólo en lo atinente a la consolidación de una cultura de paz y construcción de ciudadanía, sino también frente a las tareas pendientes de formar y empoderar a los niños, niñas y adolescentes que han sufrido directamente las consecuencias del conflicto armado, así como la atención integral de sus necesidades básicas insatisfechas y el restablecimiento de sus derechos.

En segundo término, se torna necesario determinar las probabilidades de cumplimiento de los derechos, obligaciones y metas contemplados en el Sistema de Convivencia Escolar previamente señalado, a sabiendas de que el propio Gobierno Nacional reconoce que los recursos para financiar y concretar la Ley 1620 son limitados, por no decir nulos, tal como quedará evidenciado en el desarrollo de este trabajo. De este modo, la clase dirigente espera que la realidad ceda ante los propósitos de la señalada norma, no obstante su ineptitud para ser eficaz.

En ese orden de ideas, las preguntas que surgen y que se buscan responder a lo largo de éste escrito son las siguientes: ¿En qué medida el Sistema de Convivencia Escolar integra y visibiliza a la niñez y a la juventud marginada de nuestro país, en un escenario de post-acuerdo?, ¿Son suficientes las líneas de acción contempladas en dicho marco normativo para resolver las necesidades económicas de este grupo poblacional, restablecer sus derechos prevalentes y materializar una cultura de paz, teniendo en cuenta los contextos sociales y culturales?, ¿Cuáles son las políticas 
públicas del orden nacional que desarrollarán los propósitos contemplados en la Ley 1620?, Y finalmente, ¿Es el Sistema de Convivencia Escolar un saludo a la bandera, una mera enunciación normativa en torno a diferentes materias que ya habían sido reguladas?

Para abordar los anteriores interrogantes, primero se explicará la magnitud del acoso escolar durante los últimos años a nivel mundial y en el ámbito nacional, así como sus diferentes implicaciones en los estudiantes. Luego, se hará una caracterización general del Sistema de Convivencia Escolar anteriormente referenciado. Posteriormente, se determinará la efectividad de las disposiciones prescritas en la Ley 1620. Y, por último, se analizará la función latente de dicha norma, es decir, su eficacia simbólica y su capacidad para crear ciertas representaciones sociales, lo cual nos permitirá comprender la verdadera intención de gobernar basado únicamente en el acto de promulgación de esa Ley, esperando que ésta por si sola modifique y transforme el statu quo.

\section{Una aproximación a la problemática}

Son varios los tipos de violencia y discriminaciones que deben enfrentar los niños, niñas y adolescentes al interior de la escuela, de la familia y de la comunidad, los cuales obedecen a múltiples causas y tienen repercusiones muy delicadas en el estado físico, mental y emocional de cada uno de ellos(as). Por lo tanto, las variaciones en la prevalencia de la vulneración, así como en las formas de victimización experimentadas en diferentes edades o cursos académicos puede atribuirse a las características individuales o contextuales presentes en las diferentes etapas de su desarrollo (Babarro, Ruiz y Martínez, 2014, p.8).

En el contexto nacional e internacional, desde hace varias décadas son cada vez más notorios y preocupantes los casos de acoso escolar, en los cuales resultan afectados 
los niños(as) y jóvenes a quienes se les vulnera su proceso de formación, su integridad y sus posibilidades de relacionarse con el otro, desde un plano horizontal y de respeto mutuo. De hecho, podría pensarse que esta problemática la padecen los países marginados o con graves dificultades en el orden político, social, económico o cultural. No obstante, la anterior es una situación grave que está presente en todos los países del mundo, incluidos los industrializados y emergentes, quienes han emprendido diferentes campañas de sensibilización, leyes y proyectos para reducir los correspondientes indicadores, sin obtener mayor éxito, salvo algunas excepciones.

Para ilustrar, alrededor del $22 \%$ de los estudiantes de Estados Unidos, entre las edades de 12 y 18 años, informaron que fueron acosados en la escuela durante el año escolar del 2013, lo cual significa que más de dos (2) de cada diez (10) estudiantes están siendo intimidados en los establecimientos educativos (Wallace, 2015). La problemática se agudiza en este país debido a que muchos menores que padecen algún tipo de acoso escolar o bullying, apelan a las armas como respuesta frente a las amenazas o agresiones a las que son sometidos, generando un desenlace que resulta peor de lo imaginado, al cobrar la vida de cientos de niños, niñas y adolescentes.

Paralelamente, en Francia el $40 \%$ de los niños ha sufrido alguna vez algún tipo de hostigamiento. Específicamente, el bullying perjudica alrededor de 1,4 millones de alumnos, uno (1) de cada diez (10) niños en la escuela primaria y secundaria lo han experimentado, lo cual puede ir desde el simple robo de la merienda hasta casos de insultos, intimidación, amenazas, palizas, extorsión o violencia sexual. Ahora bien, según el Ministerio de Educación de este país, el acoso cibernético es el más frecuente en dicho grupo poblacional, el cual se manifiesta en el envío de mensajes humillantes o insultantes que afecta a uno (1) de cada cinco (5) alumnos, seguido de 
llamadas telefónicas amenazantes, robos de identidad digital, entre otros tipos de acoso (Bellver, 2013).

Por su parte, en Japón hay una serie de registros que indican que muchos estudiantes deciden suicidarse en el inicio de cada periodo académico (Abril y Septiembre de cada año). En efecto, entre 1972 y 2013, de los dieciocho mil cuarenta y ocho (18.048) niños que se suicidaron, noventa y dos (92) lo hicieron el 31 de agosto, ciento treinta y uno (131) se quitaron la vida el primero de septiembre, y noventa y cuatro (94) lo realizaron el segundo día de éste último mes. Se estima entonces que el número de personas afectadas por este fenómeno es de setecientos mil (700.000) o incluso más. Tan es así, que se ha declarado el 1 de Septiembre como el día más negro de ese país, poniendo de presente las circunstancias complejas que afrontan diferentes alumnos japoneses como consecuencia del acoso escolar, especialmente al inicio de cada curso académico (Kremer y Hammond, 2013).

Entre tanto, en Colombia según un estudio realizado por la Fundación Plan en seis departamentos del país, el $22,5 \%$ de los menores han ejercido algún tipo de violencia y el $56,8 \%$ se reconocen como víctimas de 'bullying'. Además, el estudio manifiesta que el acoso escolar está asociado con una serie de representaciones sociales relacionadas con la definición de ser "avión", entendiendo por tal ser "avispado", no dejarse de los demás, y ver cómo sacar provecho del otro. Igualmente, sobre los tipos de violencia escolar frecuentados, se encuentra que en el caso de las mujeres son muy comunes las amenazas (29,5\%), mientras que en los niños lo son las agresiones físicas (40 \%) (Redacción de El País, 2014).

Estos datos son relevantes porque evidencian la magnitud del problema que se está analizando. Sin embargo, tan sólo son la punta del iceberg, en la medida en que no Revista Legem, Universidad del Atlántico 4 (2), Julio - Diciembre 2018, pp. 21-51 
reflejan otras variables que ocurren en la práctica, como por ejemplo la participación y re-incidencia de los compañeros de clase en los tipos de violencia escolar, así como las agresiones que se presentan por fuera del establecimiento educativo. Tampoco se sabe con exactitud los correspondientes sub-registros, es decir, las cantidades reales de niños y jóvenes que a pesar del acoso que padecen no son inscritos en las estadísticas, bien sea porque poseen un menor rango de edad al comprendido en los diversos estudios realizados, o, a razón de que los estudiantes no informan del bullying al que están siendo sometidos, como sucede en muchos de los casos.

A continuación se planteará una descripción sucinta del acoso escolar y sus diferentes implicaciones sobre los niños, niñas y adolescentes, con la finalidad de comprender los efectos que genera dicha violencia escolar, así como la complejidad de las diferentes formas de victimización. Lo anterior, resulta relevante ya que la Ley 1620 y su Decreto Reglamentario pretenden regular esta problemática (entre otros asuntos), al punto que ha sido mal denominada como Ley Anti-bullying, término que per se resulta insuficiente para entender los componentes que abarca el Sistema de Convivencia Escolar.

\subsection{Consecuencias e implicaciones del Acoso escolar}

El acoso escolar o bullying es un tipo de comportamiento agresivo, repetitivo y sistemático al que se encuentran sometidos muchos alumnos en el entorno escolar y fuera de éste, por parte de uno o más compañeros. Es una de las formas de violencia más comunes y extendidas en el contexto de la escuela que implica una relación asimétrica de dominio-sumisión, caracterizada por un desequilibrio de poder entre el acosador y la víctima (Babarro, et al., 2014). Dichas características (acoso reiterado y relación de poder de dominio-sumisión) son las que permiten distinguir el bullying de otras acciones violentas en el entorno escolar, en tanto que se atenta contra la 
dignidad del niño(a) y del joven, les genera graves secuelas emocionales, sociales, psicológicas y físicas, y supone una constante perturbación en las relaciones entre estudiantes.

Este fenómeno presenta diferentes formas de discriminación y agresión física, verbal, social, relacional, dentro de las cuales, cabe resaltar el aumento significativo que ha tenido en los últimos años los acosos cibernéticos o ciberbullying, a través de las redes sociales o mediante la dispersión de textos e imágenes por correo electrónico, entre otros, suscitando nuevas formas de agresión que resultan provechosas para los acosadores ante la posibilidad del anonimato y que van más allá del contexto espaciotemporal del aula de clases, pero cuyos efectos se extienden al entorno escolar.

Ahora bien, ciertos tipos de victimización son menos frecuentes que otros, y, debido a su continuidad, son relativos, ya que cambian con el transcurso de la edad y dependen del género del estudiante. Así, tenemos que la frecuencia de la victimización social, comparada con la victimización física, es menor durante la preadolescencia y se incrementa paulatinamente durante la adolescencia (Babarro, et al., 2014). No obstante, en el caso de las chicas, el acoso se manifiesta y persiste en formas más encubiertas y de exclusión relacional que en comparación con los niños, los cuales emplean habitualmente agresiones físicas como respuesta frente al acoso escolar.

Postigo y colaboradores (2013) describen la trayectoria que sigue un niño, una niña, o un joven víctima del acoso escolar, tomando las fases propuestas por Leymann (1996) sobre el acoso laboral. El fenómeno comenzaría por una fase inicial en la que la víctima comienza a ser progresivamente acosada por el agresor. Ante estos ataques, si la víctima responde sumisamente o no cuenta con una red de apoyo, se 
convierte en víctima como tal, pasando a una segunda fase llamada de latencia, en la que la relación asimétrica de fuerza y poder entre el acosador y la víctima adquiere un mayor relieve. Finalmente, durante la tercera fase, también denominada de estigmatización, el acoso ha quedado establecido, al punto de que la víctima comienza a identificarse como tal dentro del grupo (citado en Babarro, et al., 2014).

Cabe anotar que, en el grupo poblacional previamente señalado se adoptan diferentes roles durante las situaciones de acoso, en la medida en que existen altos porcentajes de la población estudiantil que siendo víctimas también desempeñan el rol de acosadores, posiblemente con estudiantes más débiles o vulnerables (Cepeda y Caicedo 2012), convirtiéndose en un estatus difícil de modificar debido a la intensificación de las agresiones con el transcurrir de los cursos académicos, siguiendo las fases de acoso explicadas previamente.

En ese sentido, la victimización prolongada conlleva una serie de consecuencias negativas en los estudiantes, e incluso, algunas de ellas persisten por el resto de sus vidas si no son tratadas a tiempo. En general, las causas que generan los síntomas físicos, psicológicos y emocionales no son detectados oportunamente, tales como:

\author{
-Aislamiento \\ -Soledad \\ -Deficiente rendimiento académico
}

-Abandono escolar

-Suicidio.

-Ansiedad

-Depresión 


\section{-Estrés}

-Deseos de venganza

-Miedo

-Apatía

-Rabia reprimida

-Dolores de cabeza o estómago

-Disturbios del sueño

-Pérdida del apetito

Por ende, se ha demostrado que el acoso escolar es determinante en el desempeño académico de los estudiantes, al ser considerado como un factor psicológico negativo que afecta no sólo su rendimiento y aprendizaje, sino que además vulnera su salud mental y bienestar psicológico (Ferrel, Vélez y Ferrel, 2014). Por lo cual, no es posible hablar de una política de calidad educativa sin tener en cuenta esta circunstancia, con miras a su mitigación.

Entre tanto, son diversas las investigaciones que han establecido ciertas características comportamentales relacionadas con la victimización, que están estrechamente relacionas y pueden aparecer en muchos casos de un modo concurrente, lo cual se enmarca en dos grupos de comportamientos disfuncionales: (a) comportamientos internalizantes, y (b) comportamientos externalizantes. Veamos:

a. La internalización es la tendencia a expresar la angustia de una forma introspectiva, incluyéndose en este grupo los trastornos del estado de ánimo (i.e. trastorno depresivo mayor, distimia) y los trastornos de ansiedad (i.e. 
trastorno generalizado de ansiedad, fobias, trastorno obsesivo compulsivo, etc.).

b. La externalización describe la inclinación a expresar la angustia hacia fuera. Se consideran trastornos de tal índole el trastorno por déficit de atención e hiperactividad (TDAH), el trastorno negativista desafiante (TND), el trastorno de personalidad antisocial, así como los trastornos derivados del consumo de sustancias psicoactivas (Cosgrove, Citado en Babarro, et al., 2014).

Crick y Bigbee (1998) hallaron que la victimización relacional contribuía con mayor claridad a la aparición de trastornos internalizantes, mientras que la victimización física ejercía una mayor influencia en la aparición de los trastornos externalizantes. Igualmente, se considera que estos comportamientos pueden llegar a desencadenar problemas de comportamiento antisocial e incluso conductas delictivas.

En un estudio sobre los desajustes mentales de las víctimas de acoso escolar, un grupo de psicólogos concluyó que hay un mayor desajuste psicológico asociado a la victimización durante la etapa de educación primaria en comparación con la etapa secundaria. Esto indica la imperiosa necesidad de actualizar los manuales de convivencia y protocolos de actuación orientados a la prevención en el acoso escolar durante esta etapa educativa, entre otros mecanismos de intervención.

\section{Breve caracterización del Sistema de Convivencia Escolar}

Teniendo presente las dimensiones tan complejas que implica el acoso escolar y sus diferentes implicaciones en los estudiantes, se torna indispensable profundizar en la 
respuesta que adoptó el Estado colombiano para hacer frente a esta problemática, entre otras relacionadas, como lo es la Ley 1620 y su Decreto Reglamentario 1965 del 2013.

En efecto, dicha normatividad contempla un Sistema de Convivencia Escolar, que abarca un conjunto de acciones y procedimientos delimitado fundamentalmente a cinco ejes: construcción de competencias ciudadanas, formación para el ejercicio de los derechos humanos, sexuales y reproductivos, mitigación del acoso escolar o bullying en sus diferentes manifestaciones, reducción del embarazo precoz en la adolescencia y disminución de las enfermedades de transmisión sexual.

Lo anterior, va enfocado a los niños, niñas y adolescentes pertenecientes a la comunidad educativa en los niveles de preescolar, básica y media, a través de la puesta en marcha de cuatro grandes procesos:

a. La articulación de programas, actividades y mecanismos por parte de los establecimientos educativos, y en menor proporción, de los diferentes actores del Sistema para que fortalezcan las líneas estratégicas señaladas previamente;

b. La implementación de las rutas de atención para la convivencia escolar, la cual define los procesos y protocolos que deben seguir las entidades que conforman el Sistema de Convivencia Escolar, basada en los componentes de promoción, prevención, atención y seguimiento.

c. La actualización y ajuste de los manuales de convivencia y de los proyectos educativos institucionales según los parámetros establecidos en la Ley 1620 y su Decreto Reglamentario 1965 de 2013, incluyendo las rutas de atención 
integral y sus respectivos protocolos, los cuales deben actualizarse cada dos años como resultado de las evaluaciones que se realicen sobre su funcionamiento;

d. La adopción del Sistema de Información Unificado, que permite el registro y seguimiento de los casos de vulneración de los derechos sexuales y reproductivos, violencia y acoso escolar que afecten a los estudiantes en los establecimientos educativos.

De igual manera, este Sistema establece una estructura tripartita de comités de convivencia escolar divididos por niveles (Nacional, Territorial y Escolar), los cuales a su vez están conformados por diversas instituciones del Estado y de la sociedad civil, encargados de la coordinación de políticas, del fortalecimiento de proyectos pedagógicos y, en general, del desarrollo de las metas trazadas en la Ley 1620, consignados según las diferentes competencias.

Ahora bien, los comités escolares de convivencia tienen una conformación y funciones distintas con respecto al Comité Nacional, Departamental, Distrital y Municipal, puesto que son aquellos quienes deben conocer en primera instancia las situaciones que afecten la convivencia escolar y, por ende, son los llamados a liderar los procesos y estrategias dentro de sus correspondientes establecimientos educativos, razón por la que, estos últimos se limitan a ejercer un rol de acompañamiento, control y vigilancia.

De ahí que, las situaciones de alto riesgo de violencia escolar o de vulneración de derechos sexuales que no se puedan resolver por las vías contempladas en el manual de convivencia de cada colegio, serán trasladadas por el Rector -según las instrucciones del Comité escolar-, al ICBF, a la Comisaría de Familia, a la Personería 
Municipal o a la Policía de Infancia y Adolescencia, dependiendo del grado de intervención que se requiera para atender cada caso en particular.

En ese sentido, mientras que el comité nacional o los comités departamentales, distritales o municipales están conformados por el respectivo titular de la cartera de salud, educación, cultura y gobierno, el director del ICBF, el Personero y el Defensor del Pueblo según corresponda -entre otros actores-, el comité escolar de convivencia está representado por el rector del establecimiento educativo, el personero estudiantil, el docente con función de orientación, el presidente del consejo de familia y demás sujetos establecidos en la señalada Ley.

Entre tanto, la participación de la familia -al igual que de la sociedad-, se reconoce en el maco de ciertas obligaciones, dentro de las cuales se resalta el acompañamiento permanente que deben tener con sus hijos en el proceso pedagógico que adelante el establecimiento educativo en torno a la convivencia y la sexualidad, el aprovechamiento del tiempo libre para el desarrollo de competencias ciudadanas, el seguimiento y la utilización de la Ruta de Atención Integral cuando se presente un caso de violencia que se encuentre tipificado en la Ley 1620 , entre otros deberes.

En materia de orientación escolar, el Sistema de Convivencia escolar prevé alianzas estratégicas entre el establecimiento educativo y las instituciones de educación superior, con el fin de que los estudiantes de último semestre de psicología, psiquiatría y programas afines puedan adelantar sus prácticas en los colegios, en el área de orientación escolar. No obstante, el Gobierno Nacional no promueve ningún incremento real de pedagogos especialistas en orientación escolar, en la medida en que se consagra la misma cantidad de la que venían disponiendo los establecimientos educativos con anterioridad a la entrada en vigencia de la mentada Ley, remitiendo, 
de este modo, a los procedimientos burocráticos existentes para realizar modificaciones o aumentos en las plantas de cargos del personal docente.

Lo mismo sucede con la atención en salud mental a los niños, niñas y adolescentes afectados por la violencia escolar o por la vulneración de sus derechos sexuales y reproductivos, en tanto que esa responsabilidad se le endilgó exclusivamente al Sistema de Salud, con base en el POS actual, sin tener en cuenta ningún criterio diferencial, ni los frecuentes obstáculos que ponen las EPS para resolver los problemas de sus usuarios, ni mucho menos la complejidad de las secuelas e implicaciones que afectan a dicho grupo poblacional, tal como se explicó en el punto anterior.

Para finalizar esta caracterización, es importante señalar que con la aprobación de esta Ley y su Decreto reglamentario, el Gobierno estableció una estrategia de zanahoria y garrote bastante desbalanceada, ya que mientras por un lado únicamente promueve, a manera de reconocimiento, la participación de las cinco mejores propuestas de mejoramiento de convivencia escolar por parte de los establecimientos educativos en un foro educativo nacional, por el otro, dispuso de diferentes sanciones disciplinarias y amonestaciones públicas frente al retraso o incumplimiento de las funciones asignadas por el Sistema de Convivencia a las instituciones educativas, a los docentes y a los directivos, con lo cual exoneró, de manera sofisticada, las tareas y compromisos del Gobierno, del Ministerio de Educación y demás entidades del Orden Nacional en lo que respecta a la financiación e implementación de los objetivos estipulados.

\section{El Sistema de Convivencia Escolar es más de lo mismo: Enunciación normativa, poca efectividad}


Como podemos observar, son varias las pretensiones del Sistema de Convivencia Escolar, pero no por ello resultan novedosos. De hecho, la mayoría de estas disposiciones tienen su origen en la Ley 115 de 1994 (más conocida como Ley General de la Educación), así como en la Ley 1098 de 2006 (Código de la Infancia y la Adolescencia), entre otras normas. Tanto es así que en un coloquio organizado por la Universidad Eafit en el 2013 sobre la implementación de la Ley 1620, un Delegado del Ministerio de Educación Nacional reconoció textualmente que dicha normatividad "no trae nada nuevo", sino que simplemente "retoma muchas cosas que estaban absolutamente perdidas, que estaban puestas en el anaquel sin que hubiera una respuesta del sector educativo, ni tampoco de otros sectores, en esa necesaria corresponsabilidad que tiene que existir para estos propósitos" (Varela, 2013).

En efecto, algunos de esos objetivos de vieja data que ha promulgado el Estado colombiano al interior de las escuelas han consistido en "fomentar en la institución educativa, prácticas democráticas para el aprendizaje de los principios y valores de la participación y organización ciudadana y estimular la autonomía y la responsabilidad" (Ley 115 de 1994, artículo 13, literal c), "desarrollar una sana sexualidad que promueva el conocimiento de sí mismo y la autoestima, la construcción de la identidad sexual dentro del respeto por la equidad de los sexos, la afectividad, el respeto mutuo y prepararse para una vida familiar armónica y responsable(ibídem literal d)"; "investigar y sancionar severamente los delitos en los cuales los niños, las niñas y las adolescentes son víctimas, y garantizar la reparación del daño y el restablecimiento de sus derechos vulnerados(Ley 1098 de 2006, artículo 41, numeral 6)", "prevenir y atender la violencia sexual, las violencias dentro de la familia y el maltrato infantil, y promover la difusión de los derechos sexuales y reproductivos"( Ley 1098 de 2006, artículo 41, numeral 26), y la obligación de que los miembros de éste grupo poblacional "serán protegidos contra la transmisión del VIH-SIDA y las infecciones de transmisión sexual"( Ley 1098 de 2006, artículo 20, numeral 18). 
En estas circunstancias, la Ley 1620 y su Decreto Reglamentario son una condensación de diferentes asuntos que perjudican la integridad de los niños, niñas y adolescentes, y que deterioran la convivencia escolar, los cuales han sido regulados previamente y se encontraban dispersos en el Ordenamiento Jurídico de nuestro país, pero cuyos niveles de incidencia sobre las problemáticas que pretendían resolver han sido insuficientes desde entonces, por lo cual, no dejaron de ser letra muerta.

Ahora bien, a la citada Ley se le añadieron ciertos órganos, pautas y responsabilidades que en principio plantean la posibilidad de que, esta vez, se está haciendo algo diferente $\mathrm{y}$, por consiguiente, el fantasma del fracaso de las veces pasadas estaría descartado por el momento. No obstante, si bien los preceptos que contiene dicha Ley constituyen avances en lo referente a la actualización de los manuales de convivencia, de las rutas de atención y de los enfoques de derechos, dichas disposiciones no garantizan, en modo alguno, su efectividad, ya que durante los últimos años ha quedado claro que no existe una correspondencia entre las conductas prescritas por esa norma y la realidad social.

Así lo demuestran las cifras, las cuales no sólo reflejan una situación crítica de violencia escolar -más del $50 \%$ ha sido víctima de este flagelo en Colombia, tal como se mencionó anteriormente-, sino que además evidencian un aumento significativo del maltrato infantil, de la violencia sexual, de las infecciones de trasmisión sexual y del embarazo precoz, con posterioridad a la entrada en vigencia de la Ley, a pesar de las obligaciones y sanciones establecidas en esa normatividad.

Precisamente, en el año 2015 el Instituto Colombiano de Bienestar Familiar informó que a corte de diciembre, se acumularon veintiún mil seiscientos sesenta y seis (21.666) procesos de protección por maltrato infantil, que en su mayoría sucedieron 
dentro del hogar. Por esta situación, siete mil setecientas treinta y dos (7.732) menores de edad víctimas de maltrato físico, psicológico y de negligencia fueron cobijados en dichos procesos, lo cual representa un aumento de setecientos cincuenta (750) casos adicionales de maltrato con respecto al 2014 donde se registraron seis mil novecientos ochenta y dos (6.982) casos, representando de esta manera un incremento del 9\% (Ávila, 2016).

Entre tanto, en Colombia cada día ciento veintidós (122) niños, niñas y adolescentes son víctimas de violencia sexual (Redacción de El País, 2014). Durante el año 2014, se registraron veintiún mil ciento quince (21.115) exámenes médico-legales por presunto delito sexual con un aumento de trescientos setenta y seis (376) casos en relación con el año 2013, es decir, un incremento del 1,81\%, siendo la niñez y la juventud los más perjudicados con el $85,08 \%$ de los casos reportados, según lo afirma el Instituto Nacional de Medicina Legal y Ciencias Forenses en el informe 'Forensis 2014: datos para la vida' (Velasco, 2014).

Tal como se reconoce en el señalado informe: "El fenómeno de la violencia sexual es una realidad que hace parte de la cotidianidad de la sociedad colombiana, está presente en la mayoría de contextos de violencia, sin importar las circunstancias... La violencia sexual es un fenómeno que estigmatiza a las víctimas, pero, adicionalmente, en algunos casos las hace invisibles para el Estado, negándoles la posibilidad de recibir justicia y la restitución de sus derechos... (Ibíd., p. 278)". Cabe anotar que los autores de estos crímenes siguen siendo predominantemente adultos del entorno familiar de las víctimas, esto es, padres, tíos, primos, padrastros.

En materia de embarazo precoz, Colombia ocupa el tercer lugar en Latinoamérica con la tasa más alta, después de Venezuela y Ecuador, pero esto no constituye ningún 
descubrimiento, puesto que se trata de una problemática que desde hace décadas varias va en aumento. De acuerdo al Departamento Nacional de Planeación, el $19.5 \%$ de niñas y adolescentes entre las edades de los 10 y 19 años está en embarazo (Mejía, 2015). Por su parte el ICBF afirma que desde el año 2008 hasta el 2013 han nacido cada año en promedio ciento cincuenta y nueve mil seiscientos cincuenta y seis (159.656) niños y niñas. Por lo que, una de cada cinco adolescentes entre los 15 y 19 años es madre, lo fue o está embarazada, e incluso hay algunas zonas del país donde las cifras son considerablemente mayores, como en el Cesar, Casanare, Guaviare, Huila y Magdalena (Redacción El Espectador, 2014).

En lo concerniente a las enfermedades de transmisión sexual, el Ministerio de Salud reportó un promedio anual de noventa y ocho mil cuatrocientos veintitrés (98.423) casos de ETS y ciento setenta mil (170.000) de VIH/Sida, de los cuales más del 50\% corresponde a mujeres y hombres menores de veinte (20) años, lo que no solamente refleja la gravedad del asunto, sino que pone en evidencia el desconocimiento que padece esta población sobre los signos, síntomas y formas de prevención de estas infecciones (Ministerio de Salud, 2012).

\subsection{La miopía de la Ley 1620 ante la realidad compleja del conflicto armado}

En un escenario de post-acuerdo como el que se propone el Gobierno Nacional con las FARC y el ELN, la inclusión a la sociedad civil de los niños, niñas y adolescentes que participan directamente del conflicto armado interno o de aquellos que han sido perjudicados como consecuencia de los diversos tipos de violencia, supone un reto mayor para el Estado colombiano en contextos complejos y de exclusión social, ya 
que éste último debe ofrecer las condiciones necesarias para garantizar la educación de dicho grupo históricamente abandonado, la mejora de sus condiciones de vida, el restablecimiento y la reparación de sus derechos de un modo eficiente, con miras a garantizar una convivencia sana entre estudiantes en condiciones de equidad.

Ciertamente la niñez y la juventud son las víctimas más vulnerables del conflicto armado, especialmente en la población desplazada, lo que conlleva a que sufran pérdidas irreparables de etapas fundamentales en el proceso de crecimiento. Por lo cual, tienen afectaciones psicosociales, sufren desnutrición y desprotección, no cuentan con opciones para desarrollar sus capacidades, habilidades y potencialidades, ni tampoco posibilidades de recreación o participación. Por su parte, las niñas están más expuestas a situaciones de abuso y explotación sexual, al trabajo doméstico y al abandono escolar, en la medida en que terminan asumiendo el cuidado de los hermanos (Luna y Uribe, 2015).

En ese orden de ideas, el Sistema de Convivencia escolar ésta pensado para un país que no tiene la obligación política y moral de mitigar todas esas problemáticas, ya que independientemente de las referencias que hace la norma relacionados con la construcción de ciudadanía o de una cultura de paz, no se establecen compromisos concretos por parte del Estado de atender las penurias económicas, así como reparar las diversas secuelas de las víctimas menores de edad que han sido violentados sistemáticamente durante el transcurso de la guerra, lo cual requiere un criterio diferenciador con respecto a los demás niños y jóvenes, y una intervención integral dada su situación de extrema vulnerabilidad. Tampoco se articulan políticas públicas a corto, mediano y largo plazo que busquen solventar las necesidades materiales insatisfechas de este grupo poblacional. 
En consecuencia, el Gobierno Nacional simplemente se conformó con expedir y reglamentar una norma, ignorando las dificultades de millones de niños, niñas y adolescentes que han sido directamente afectados por el conflicto armado y que requieren un enfoque integral y una atención diferencial, incluso control judicial (Pérez Vásquez 2015), los cuales no serán resueltos ni por los establecimientos educativos, ni por las entidades territoriales, ni mucho menos por las EPS, si no hay intervenciones planificadas y articuladas de los diferentes actores que conforman el Estado colombiano.

En efecto, la Ley 1620 introduce varias muletillas retóricas en torno a la eventualidad de que los diferentes sujetos que conforman el Sistema de Convivencia escolar desarrollen programas, proyectos y estrategias sobre los objetivos planteados. Sin embargo, al momento de designar competencias y responsabilidades, se establece una carga desproporcionada a los establecimientos educativos, a los docentes y a los directivos.

En cuanto a las entidades territoriales, esta normatividad reafirma lo que ya existe, es decir, que éstos pueden modificar o eventualmente incrementar los docentes orientadores con cargo al Sistema General de Participaciones, siempre y cuando cumplan con los procedimientos y requisitos burocráticos señalados para tal fin, los cuales -dicho sea de paso- son engorrosos, ya que dependen del aumento de la matrícula de los establecimientos educativos estatales, entre otras condiciones taxativas.

Como si no fuera suficiente esa distancia entre la norma y la realidad, la Ley señalada no estipula una financiación real de parte del Gobierno, ni del Ministerio de Educación, ni los demás Ministerios intervinientes, así como tampoco asigna un porcentaje del 
presupuesto nacional para cumplir con las finalidades planteadas explícitamente. De hecho, en el coloquio sobre la puesta en marcha de esa norma, el mismo delegado del Ministerio de Educación referenciado previamente, ante las preguntas: ¿Cuánto cuesta la implementación de dicha Ley? y ¿Está previsto dotar a las escuelas de nuevos recursos para cumplir con los objetivos trazados?, reconoció lo siguiente:

En la construcción de un marco legislativo, lo último que se piensa es en los recursos, en como ésto va a tener un impacto fiscal para el funcionamiento de los establecimientos educativos y para el sector educativo en general. Evidentemente el Ministerio, frente a este tema, ha puesto un especial acento en lo que tiene que ver con la ampliación de la planta de los docentes orientadores. Si ustedes preguntan a cualquier persona en el Ministerio si ¿eso es suficiente?, si ¿eso es lo que va a ayudarnos a solucionar esto?, y, si ¿esos son todos los recursos que necesitaría esta Ley para entrar en funcionamiento, para responder a los retos y responsabilidades que plantea? la respuesta es definitivamente no... En nuestro país la política de educación de calidad tiene un lado muy débil, y, es el tema del financiamiento: ¿Cómo esto se apalanca con unos recursos que realmente lleguen al nivel del establecimiento educativo?... (Varela, 2013).

Para concluir este punto, dicho funcionario añadió: "Esta Ley lo que nos va a permitir, con miras a un próximo gobierno, es cómo ponerle las herramientas y recursos que necesita el establecimiento educativo para estos desarrollos" (Ibíd.).

Ahora bien, podrá decirse que en la Ley 1620 se estipuló que dichos entes territoriales pueden disponer de su presupuesto y gestionar mediante alianzas público-privadas o interadministrativas para lograr los cometidos prescritos, pero cuando analizamos en detalle lo que ello significa, sabemos muy bien que en nuestro país esta es la típica 
excusa con la que los gobiernos de turno se desentienden de sus funciones. Basta mirar el fracaso de los programas de alimentación escolar, para corroborar la idea de que las alcaldías y gobernaciones no financiarán los proyectos ni desarrollarán las políticas públicas que se requieren para materializar los objetivos explícitos perseguidos por la Ley, mucho menos para proteger integralmente a los niños(as) y jóvenes afectados por el conflicto armado y la exclusión social.

En síntesis, no ha habido mayor impacto del texto jurídico que conforma el Sistema de Convivencia Escolar, por cuanto los resultados obtenidos hasta el momento han sido escasos, lo cual, desde un análisis de la efectividad instrumental sería atribuible a la enorme disparidad entre el contenido de la Ley y la complejidad de la realidad social que se busca regular, y ante la monumental desfinanciación para poner en marcha dicha normatividad. Sin embargo, si acudimos a la función latente de la Ley 1620, a través del estudio de su eficacia simbólica, podemos desentrañar la causa real de su inoperancia, así como el verdadero propósito que hay detrás de la promulgación de dicha norma, tal como se explicará a continuación.

\section{La Eficacia simbólica de la Ley 1620 de 2013}

Lo señalado anteriormente pone en evidencia que la intención perseguida con la expedición de esa norma no radica en su contenido explícito y, probablemente, ni siquiera se pueda encontrar en las discusiones que le dieron origen, por lo que, debemos acudir al concepto de eficacia simbólica desarrollado por Mauricio García Villegas, para entender la intencionalidad de la norma en este caso, el cual supera su carácter instrumental. 
Dos ejemplos permitirán aproximarnos a dicha noción: El primero se refiere al reconocimiento del poder de la curación del chamán, cuya credibilidad colectiva no solamente es importante para el éxito de su operación curativa, sino que la curación misma únicamente se explica por la existencia de tal reconocimiento. El segundo tiene que ver con las ceremonias indígenas de la lluvia o de la fertilidad, las cuales no producen lluvia ni aumentan los nacimientos, pero tienen la función latente, no declarada, de ofrecer una expresión colectiva a sentimientos que son fundamentales para la identidad del grupo (García, 1993).

De este modo, sabemos que en el derecho existe un tipo de eficacia que se vale de los mismos mecanismos simbólicos que le permiten al chamán tener éxito en sus operaciones curativas y les resultan funcionales a las ceremonias realizadas por los grupos indígenas. Por ende, a partir de un discurso en particular, se crea una mediación psicológica, un poder retórico, en el que la falta de correspondencia con la realidad objetiva no tiene mayor relevancia, lo importante es que la norma, al igual que la recitación chamánica y el ritual indígena, sea conocida y aceptada por los receptores. Es decir, la forma mítica prevalece sobre la veracidad del relato (García, 1993).

Tal como señala García Villegas "si se analiza el derecho como un discurso que se aplica en un espacio en el cual tienen lugar múltiples relaciones de poder, de las cuales él mismo hace parte, se puede ver como muchas de las decisiones que se toman dentro de una comunidad jurídica responden a un juego inteligente en aras del fortalecimiento de ciertos intereses y en el que se combina la más benéfica relación entre textos eficaces e ineficaces, sin que ello pueda ser explicado con base en la dificultad de las materias tratadas o en la ineptitud de los aplicadores (1993, p. 80)". 
Por esa razón, la incidencia de las normas no está restringida exclusivamente a las posibilidades de concretar o no los objetivos explícitamente trazados, sino que también tiene que ver con las representaciones colectivas que los textos jurídicos logran infundir en la sociedad. De hecho, existen muchas normas con una débil eficacia instrumental, pero con una función política determinante que no se desprende de la lectura del texto.

En ese sentido,

El derecho, como todo discurso, actúa en un campo de producción simbólica. En la realidad social se constata como los enunciados jurídicos no se limitan a comunicar una información sobre el deber ser social. El derecho, como todo lenguaje, sirve para hacer cosas diferentes de las que él mismo dice querer hacer. El derecho ordena, forma e informa no solamente a través del sentido explícito de sus textos y ritos, sino también a través de su misma producción y de su propio consumo... La fuerza del derecho se encuentra en su carácter de discurso legal y discurso legítimo, en su capacidad para crear representaciones de las cuales se derive un respaldo político; en su aptitud para movilizar a los individuos en beneficio de una idea o una imagen, con independencia de la evaluación de veracidad o de efectividad que pueda hacerse de la correspondencia de dicha idea o imagen con la realidad (García, 1993, p.87).

Teniendo en cuenta esta perspectiva, resulta posible enmarcar la eficacia simbólica de la Ley 1620 en el modelo de eficacia enunciativa desarrollada por el autor en comento, en la medida en que los objetivos instrumentales y conductas relevantes prescritas en dicha norma, no logran aplicarse ni traducirse en la realidad social. No obstante, con el sólo acto de promulgación, el Gobierno y el poder político dominante que actúan en su nombre y que padecen una crisis de legitimidad, buscan sacar el mayor provecho coyuntural de la existencia del texto jurídico, válido e ineficaz a la vez. 
Precisamente, el profesor García Villegas explica un subtipo de la eficacia enunciativa, con el que se puede comprender la apropiación política del fenómeno jurídico de la promulgación para obtener los beneficios de la eficacia simbólica que trae la publicación de nuevas normas, a la que denomina eficacia "performativa", a saber:

En el ordenamiento jurídico existen normas que son creadas con el único objetivo de ser promulgadas, de ser enunciadas, y no con el de ser aplicadas. El poder del derecho es el poder de decir el derecho... En estos casos, la promulgación de las norma, más que un instrumento para comunicar una decisión proveniente del poder, se convierte en un poder que se ejerce con la sola promulgación. El poder del espectáculo que comunica nuevas formas hay que buscarlo en lo espectacular de este poder y no en el contenido de las nuevas normas... La promulgación sirve entonces de canal de comunicación entre un sistema social productor de demandas y un sistema político llamado a dar respuesta a estas demandas. Así la norma se convierte en una respuesta política, derivada del orden "hacer creer", y no del orden del "hacer hacer", tal como se pretende desde un punto de vista dogmático- jurídico (1993, p.85).

Por lo tanto, la verdadera pretensión de la Ley 1620 es la obtención de legitimidad por parte de la clase dominante de nuestro país, a través de los beneficios puramente simbólicos que genera la promulgación de la norma, en el cual, el desconocimiento y el grado de complejidad de los asuntos que busca regular, la falta de recursos que requiere su implementación o la exigüidad de los mecanismos establecidos para su ejecución no constituyen las causas del fracaso instrumental de dicho escrito jurídico, sino que su inaplicación es un resultado previsto desde su misma iniciativa. 
De este modo, la promesa de que en un futuro algún gobernante asignará los recursos requeridos para la implementación de la señalada Ley, se convierte en un elemento distractor que contribuye a la estrategia de la eficacia simbólica utilizada por el Gobierno, que de momento sirve para satisfacer las expectativas de los ciudadanos en espera de la eventual implementación de dicha norma, antes que para materializar los objetivos prescritos textualmente, pero cuya confianza finalmente se terminara socavando en la conciencia de éstos, al término de un periodo prolongado de tiempo.

Parafraseando a García Villegas (1993), el asunto no es que la Ley 1620 choque con la realidad que se resiste al cambio y por eso resulta ineficaz; es más bien que la realidad no cambia porque choca con la pretensión latente de la norma, la cual persigue por sí misma la ineficacia.

\section{Conclusiones}

En Colombia hay necesidades insatisfechas y problemáticas del orden social, económico, político y cultural que tornan imperiosa la tarea de transformar la realidad de los niños, niñas y adolescentes, con miras a garantizar, entre otras, una sana convivencia escolar en condiciones de equidad, en el marco de los derechos humanos, sexuales y reproductivos. Aún más, de ese grupo en general, hay una población vulnerable en particular, los cuales han sido perjudicados directamente por el conflicto armado y, por ende, requieren un tratamiento diferencial y un enfoque integral, teniendo en cuenta el contexto de cada víctima, así como las consecuencias y secuelas que se han ocasionado en muchos de ellos.

Ante el fracaso de las medidas legislativas que de un modo transversal intentaron regular ciertas materias que afectan a los estudiantes en el entorno escolar, el 
Gobierno actual decidió promulgar la Ley 1620 de 2013 y su Decreto Reglamentario, los cuales asignan ciertas responsabilidades y retos asimétricos en el papel, especialmente a los establecimientos educativos, pero mantienen los mismos requisitos burocráticos para aumentar el número de pedagogos especialistas en orientación escolar, no establecen recursos para financiar su puesta en marcha, ni tampoco articulan políticas públicas concretas a corto, mediano y largo plazo que prevengan y mitiguen las problemáticas abordadas por dicha normatividad.

En ese sentido, el balance efectuado a la implementación de dicha norma, pone de presente una falta de concreción de sus objetivos explícitos, puesto que en los últimos años han aumentado en nuestro país los casos de violencia sexual, embarazo precoz, acoso escolar y transmisión de ETS. Ahora bien, lo anterior no puede atribuirse a la complejidad de los asuntos que se pretenden regular o a la falta de aplicación de los mecanismos establecidos en el Sistema de Convivencia Escolar por parte del Estado y los diferentes actores que lo conforman. Su inefectividad solo puede ser entendida a partir de la noción de eficacia simbólica, es decir, de la intención política de la clase dirigente al momento de promulgar la norma y los efectos que trajo consigo su expedición, más allá de su carácter instrumental y manifiesto.

En efecto, frente a las múltiples dificultades que padecen millones de niños, niñas y adolescentes en nuestro país, dada la gravedad de las problemáticas que afectan su integridad y formación -las cuales no se reducen a las materias que delimita la Ley-, y ante la crisis de legitimidad del poder político dominante, el Gobierno creó una Ley adornada de muletillas retóricas, cuya finalidad no es propiamente la puesta en marcha de sus disposiciones literales, sino que su función latente es la configuración de determinadas representaciones colectivas en la sociedad, que hacen creer que algo se está haciendo para contrarrestar determinados flagelos que perjudican a dicho grupo poblacional. 
Por consiguiente, en este escenario la función del Gobierno consiste en satisfacer momentáneamente las demandas y reclamos provenientes de la sociedad en torno a ciertas temáticas imperiosas, a pesar de todos los errores y omisiones denunciados a lo largo de este escrito, en el cual la aplicación de los objetivos estipulados textualmente en la Ley 1620, dependen de la estrategia empleada deliberadamente con la promulgación de la misma, la cual prevé su inefectividad desde su misma expedición.

\section{Referencias.}

Ávila, C. (4 de Febrero de 2016,). Cada día, 171 mujeres son víctimas de maltrato intrafamiliar. Periódico El Tiempo

Babarro, J., Ruiz, E., y Martínez, R. (2014). Desajuste psicológico de las víctimas de acoso escolar: Un análisis evolutivo desde la educación primaria hasta la secundaria. Colegio Oficial de Psicólogos de Madrid.

Bellver, J. (27 de Noviembre de 2013). Alarma en Francia ante el incremento del acoso escolar. Periódico El Mundo. 
Cepeda, E. y Caicedo, G. (2012). Acoso escolar: caracterización, consecuencias y prevención. Bogotá: Universidad Nacional de Colombia.

Cosgrove, V. E., Rhee, S. H., Gelhorn, H. L., Boeldt, D., Corley, R. C., Ehringer, M. A., ... Hewitt, J. K.(2011). Structure and etiology of co-occurring internalizing and externalizing disorders in adolescents. Journal of Abnormal Child Psychology, 39(1), 109-123.

Crick, N. R. y Bigbee, M. A. (1998). Relational and overt forms of peer victimization: a multiinformant approach. Journal of consulting and clinical psychology, 66, 337347.

Ferrel, F., Vélez, J., y Ferrel, L (2014). Factores psicológicos en adolescentes escolarizados con bajo rendimiento académico: depresión y autoestima. Revista Encuentros, ISSN 1692-5858. No. 2., p. 35-47.

García, M. (1993). La eficacia simbólica del derecho: examen de situaciones colombianas. Bogotá: Ediciones Uniandes.

Kremer, W., y Hammond, C (2013). "Hikikomori": por qué tantos japoneses no quieren salir de sus cuartos. BBC Mundo.

Leymann, H. (1996). The content and development of mobbing at work. European Journal of Work and Organizational Psychology, 5, 165-184.

Luna, G y Uribe, F. (2015). NIÑAS Y NIÑOS: Determinantes en la Construcción de la Paz Territorial, Estable y Duradera

Mejía, J. (2016) La realidad de los embarazos tempranos en Colombia. Semana.com. 
Ministerio de Salud (2012). Situación de las infecciones de transmisión sexual diferentes al VIH. Colombia 2009 - $2011 . \quad$ Disponible en: https://www.minsalud.gov.co/salud/Documents/observatorio_vih/documentos/m onitoreo_evaluacion/1_vigilancia_salud_publica/a_situacion_epidimiologica/SIT UACION\%20DE\%20LAS\%20INFECCIONES\%20DE\%20TRANSMISION1.pdf

Pérez Vásquez, R. (2015). La decisión judicial. Erg@omnes, 7(1), 30-55. https://doi.org/https://doi.org/10.22519/22157379.742

Postigo, S., González, R., Montoya, I. y Ordóñez, A. (2013). Theoretical proposals in bullying research: a review. Anales de Psicología, 29, 413-425.

Redacción El Espectador (22 de Septiembre de 2014). Alarmantes cifras de embarazo adolescente en Colombia. Periódico El Espectador.

Redacción de El País. (2014). Acoso escolar afecta a 77,5 \% de los estudiantes colombianos. Periódico El País.

Varela, S. (Julio 19 de 2013). Coloquio Principales retos de la implementación de la Ley 1620, ley de convivencia escolar. [Archivo de video] Disponible en: http://envivo.eafit.edu.co/EnvivoEafit/?p=2415

Velasco, V. (2014). Exámenes medicolegales por presunto delito sexual. Colombia. Forensis 2014. Datos para la vida, Instituto Nacional de Medicina Legal y Ciencias Forenses.

Wallace, K. (2015). Disminuye el 'bullying' en las escuelas de EE.UU., indica un nuevo informe. CNN 